\title{
Sensitive detection of multiple pathogens using a single DNA probe
}

\begin{abstract}
A simple but promising electrochemical DNA nanosensor was designed, constructed and applied to differentiate a few food-borne pathogens. The DNA probe was initially designed to have a complementary region in Vibrio parahaemolyticus (VP) genome and to make different hybridization patterns with other selected pathogens. The sensor was based on a screen printed carbon electrode (SPCE) modified with polylactide-stabilized gold nanoparticles (PLA-AuNPs) and methylene blue (MB) was employed as the redox indicator binding better to single-stranded DNA. The immobilization and hybridization events were assessed using differential pulse voltammetry (DPV). The fabricated biosensor was able to specifically distinguish complementary, non-complementary and mismatched oligonucleotides. DNA was measured in the range of $2.0 \times 10^{-9}-2.0 \times 10^{-13} \mathrm{M}$ with a detection limit of $5.3 \times 10^{-12} \mathrm{M}$. The relative standard deviation for 6 replications of DPV measurement of $0.2 \mu \mathrm{M}$ complementary DNA was $4.88 \%$. The fabricated DNA biosensor was considered stable and portable as indicated by a recovery of more than $80 \%$ after a storage period of 6 months at $4-45^{\circ} \mathrm{C}$. Cross-reactivity studies against various food-borne pathogens showed a reliably sensitive detection of VP.
\end{abstract}

Keyword: Electrochemical DNA biosensor; Polylactide-stabilized gold nanoparticles (PLAAuNPs); Nucleic acid hybridization detection; Methylene blue; Food-borne pathogens 